\title{
Multistability in Horizontal Platform System with and without Time Delays
}

\author{
Karthikeyan Rajagopal (D), Prakash Duraisamy, \\ Riessom Weldegiorgis, and Anitha Karthikeyan \\ Centre for Nonlinear Dynamics, Defense University, Bishoftu, Ethiopia \\ Correspondence should be addressed to Karthikeyan Rajagopal; rkarthiekeyan@gmail.com
}

Received 2 September 2017; Revised 25 December 2017; Accepted 11 January 2018; Published 11 February 2018

Academic Editor: José Manoel Balthazar

Copyright (c) 2018 Karthikeyan Rajagopal et al. This is an open access article distributed under the Creative Commons Attribution License, which permits unrestricted use, distribution, and reproduction in any medium, provided the original work is properly cited.

\begin{abstract}
Chaotic behavior and bifurcation analysis of horizontal platform systems (HPS) have been investigated widely by many researchers. However, the multistable features of such systems have not been investigated, and hence we identified the multistable parameter and investigated the coexisting attractors of the HPS. To understand the effects of time delays on the nonautonomous and autonomous HPS, we introduced a constant time delay in the state feedback variable. Investigation of the bifurcation of the time delayed HPS with time delay and parameters reveals that the system behavior differs between the autonomous and nonautonomous HPS. To investigate the multistability existence in time delayed HPS, we plot the bifurcation of the autonomous HPS and show the multistability and coexisting attractors.
\end{abstract}

\section{Introduction}

Mathematical models of most of the physical systems show complex nonlinear behaviors. The superposition principle is very useful in linear analysis, which is not valid in the case of nonlinear analysis. The uncertainty with system models often demands the nonlinear dynamical models to be analyzed without linearization. Since mass, damper, and spring are the basic components of a vibratory system, nonlinearity may be introduced into the governing differential equation through any of these components. Chaos modeling has applications in many areas in science and engineering [1]. Some of the common applications of chaotic systems in science and engineering are chemical reactors, Brusselators, dynamos, tokamak systems, biology models, neurology, ecology models, and memristive devices. Chaotic phenomenon was observed in several mechanical systems [2]. Chaos may be described as bounded random like motion exhibited by deterministic nonlinear systems characterized by their sensitivity to initial conditions. Not all nonlinear systems exhibit chaos nor does chaos occur for all combinations of system parameters and initial conditions for a given system [3].
The horizontal platform system (HPS) holds a freely rotating platform about its horizontal axis especially in offshore applications. An accelerometer is placed over the platform in order to sense the displacement; accordingly, the platform is balanced by producing inverse torque to maintain the stability of HPS. Horizontal platform system (HPS) exhibits chaotic behavior on some specified conditions [4]. Chaotic motion and bifurcation analysis of a HPS under specified conditions with its dynamical response as a function of the torque is discussed in [5]. An adaptive sliding mode controller (ASMC) is designed and implemented to control the chaotic behavior in HPS with parameter uncertainties. HPS with model and parameter uncertainties with external disturbances is investigated, the chaotic behavior of the system is controlled using HPS synchronization with time varying control [6], and the dynamic analysis of a fractional order horizontal platform system (FOHPS) and its fractional order finite time chaos control design are investigated in [7].

Motivated by the above mentioned discussions, we are interested in analyzing the HPS with autonomous and nonautonomous modeling. Most of the earlier literature has discussed and analyzed the regular bifurcation pattern of the HPS, but none has presented the multistable behavior of the 


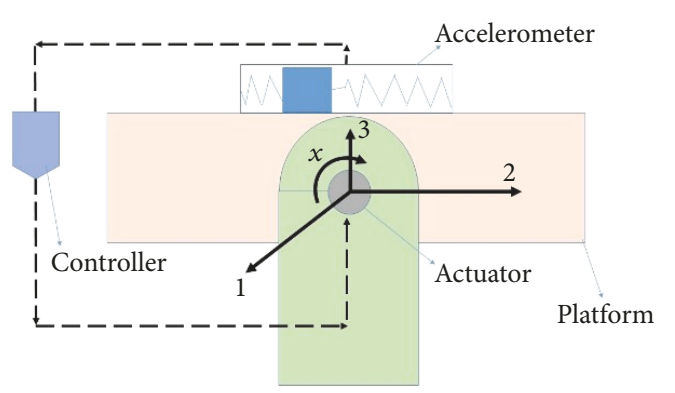

(a) Physical model of HPS

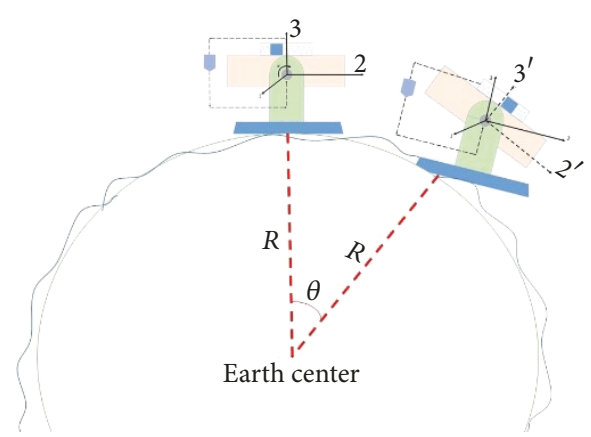

(b) HPS on earth

FIGURE 1

HPS which we have presented here. We also discuss the effect of constant time delays on the nonlinear behavior of the HPS and show the effect of time delays on autonomous and nonautonomous HPS.

\section{Mathematical Modeling of HPS}

We have used the HPS model described in [4-7] as given by Figures 1(a) and 1(b). While the platform varies from horizontal position, the accelerometer measures and sends it to the controller and the controller will produce inverse torque in order to stabilize the platform; the actuator will execute the inverse torque.

The basic assumption is that the horizontal platform rests on spherical joint which enables movement in coupled manner when it is displaced in one rotational axis. $x(t)$ represents the rotation of the platform in relation to the earth. Consider $A_{1}, A_{2}$, and $A_{3}$ are the inertia moments of the platform for axes 1,2 , and 3 , respectively. $D$ denotes the damping coefficient, $R$ represents radius of the earth, $r$ is the proportional constant of the accelerometer, $g$ denotes gravity, and $F \cos \omega t$ is the harmonic torque.

The governing law is Newton's 2nd law of motion for rotational motion:

$$
\sum M=I \ddot{x} .
$$

The net moment acting on a rigid body is equivalent to the product of the moment of inertia and angular acceleration about an axis.

$$
\sum M=A_{1} \ddot{x}
$$

When the horizontal platform is displaced with a relative angular displacement " $x$," the moments produced on the consequence are the resisting moment due to damping (fluidic friction, Coulomb friction), the moment due to accelerometer, and the coupling effect.

The damping torque is

$$
T_{d}=D \dot{x}
$$

Force acting due to accelerometer is

$$
\begin{aligned}
\cos (90-x) & =\frac{f}{m g} \\
f & =m g \cos (90-x) .
\end{aligned}
$$

The offset distance between center of mass of accelerometer and the center of mass of platform is " $a$." The accelerometer torque moment is

$$
\begin{aligned}
& M=f \cdot a \\
& M=m g a \cos (90-x) .
\end{aligned}
$$

Consider $m a=r$,

$$
M=r g \sin x .
$$

Moment equation with angular velocities in terms of Euler angles and Euler rates is

$$
L=I_{x} \dot{p}+I_{x z} \dot{r}+q r\left(I_{z}-I_{y}\right)-I_{x z} p q,
$$

where $q=\dot{\theta} \cos \phi+\dot{\psi} \cos \theta \sin \theta$,

$$
\begin{aligned}
r & =\dot{\psi} \cos \theta \cos \phi-\dot{\theta} \sin \phi \\
\theta & =\text { Pitch angle } \\
\phi & =\text { Roll angle } \\
\psi & =\text { Yaw angle. }
\end{aligned}
$$

Since $I_{x z}=0$ and $I_{x} \dot{p}=A_{1} \ddot{x}$,

$$
\begin{aligned}
L= & A_{1} \ddot{x}+q r\left(I_{z}-I_{y}\right) \\
L= & A_{1} \ddot{x}+(\dot{\theta} \cos \phi+\dot{\psi} \cos \theta \sin \theta) \\
& \cdot(\dot{\psi} \cos \theta \cos \phi-\dot{\theta} \sin \phi) \cdot\left(A_{2}-A_{3}\right) .
\end{aligned}
$$

We know that $\dot{\psi}=0$

$$
\begin{aligned}
L & =A_{1} \ddot{x}-\dot{\theta}^{2} \cos \phi \sin \phi \cdot\left(A_{2}-A_{3}\right) \\
& =A_{1} \ddot{x}-\frac{\dot{\theta}^{2} R}{R} \cos \phi \sin \phi \cdot\left(A_{2}-A_{3}\right) .
\end{aligned}
$$




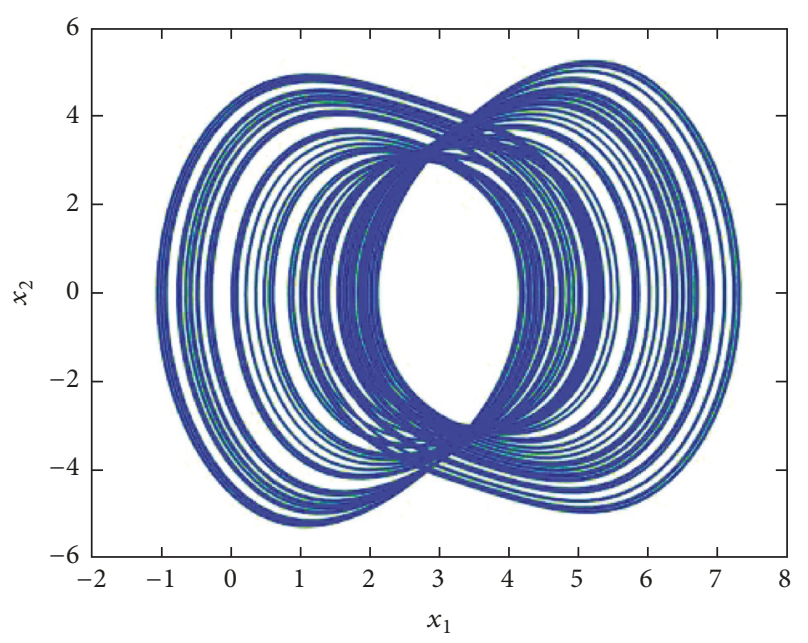

FIGURE 2: Phase portrait of the HPS.

Since $\dot{\theta}^{2} R=3 g, \phi=x$,

$$
L=A_{1} \ddot{x}-\frac{3 g}{R} \cos x \sin x \cdot\left(A_{2}-A_{3}\right) .
$$

Equation of motion can be formulated using $(* A),(* B)$, $(* \mathrm{C})$, and $(* \mathrm{D})$ :

$$
\begin{aligned}
A_{1} \ddot{x}(t) & +D \dot{x}(t)+r g \sin x(t) \\
& -\frac{3 g}{R}\left(A_{2}-A_{3}\right) \cos x(t) \cdot \sin x(t)=F \cos \omega t .
\end{aligned}
$$

Let us define the new states as $x_{1}(t)=x(t), x_{2}(t)=\dot{x}(t)$ and new parameters as $a=D / A_{1}, b=r g / A_{1}, l=\left(3 g / A_{1} R\right)\left(A_{2}-\right.$ $\left.A_{3}\right), h=F / A_{1}$, and $(*)$ can be rewritten as

$$
\begin{aligned}
\dot{x}_{1}(t)= & x_{2}(t) \\
\dot{x}_{2}(t)= & -a x_{2}(t)-b \sin x_{1}(t)+l \cos x_{1}(t) \sin x_{1}(t) \\
& +h \cos \omega t .
\end{aligned}
$$

The autonomous form of the HPS defined in (8) can be given by

$$
\begin{aligned}
\dot{x}_{1}(t)= & x_{2}(t) \\
\dot{x}_{2}(t)= & -a x_{2}(t)-b \sin x_{1}(t)+l \cos x_{1}(t) \sin x_{1}(t) \\
& +h \cos x_{3}(t) \\
\dot{x}_{3}(t)= & \omega .
\end{aligned}
$$

For $A_{1}=0.3, A_{2}=0.5, A_{3}=0.2, D=0.4, r=0.11559633$, $g=9.8, R=6378000, F=3.4, \omega=1.8$, the new parameters are solved as $a=1.333, b=3.776, l=4.6 \times 10^{-6}, h=11.333$. For initial condition $[-3.4,2.1,0]$, the HPS shows chaotic behavior. The phase portrait is shown in Figure 2.

2.1. Dynamic Analysis of HPS. In this section we analyze the dynamic properties of HPS (9) like equilibria points, Lyapunov exponents, and bifurcation diagrams. The system does not have a fixed equilibrium point as the system model is nonautonomous.
Lyapunov exponents of a nonlinear system define the convergence and divergence of the states. Lyapunov exponents (LEs) are necessary and more convenient for detecting chaos in dynamical systems, and the existence of a positive Lyapunov exponent confirms the chaotic behavior of the system [8-12]. Time series based LEs calculation methods like Wolf algorithm [9], Jacobian method [10], and neural network algorithm [11] are popularly known ways of calculating Lyapunov exponents for integer and fractional order systems. To calculate the LEs of the HPS we use the Wolf method [9] and the total simulation time taken to calculate LEs is 20000s. The LEs of the HPS are calculated as $L_{1}=0.2198, L_{2}=0$, $L_{3}=-1.5531$, and one positive LE $\left(L_{1}\right)$ confirms that HPS exhibits chaotic oscillations.

To analyze the impact of the parameters on the HPS, we derive and investigate the bifurcation plots. By fixing all the other parameters, we vary parameter $h$ between $[9.8,12.2]$, and Figures 3(a) and 3(b) show the bifurcation plots for states $x_{1}$ and $x_{2}$, respectively. The initial condition for the first iteration is fixed as $[-3.4,2.1,0]$ and is changed in every iteration as the end value of the state trajectory. The bifurcation plots show regions of period-5 limit cycle $(9.9 \leq$ $h \leq 10.05)$, period-4 limit cycle for $(11.83 \leq h \leq 11.9)$, period-2 limit cycle for $(11.91 \leq h \leq 12.1)$, and period-1 limit cycle for $(12.1 \leq h \leq 12.2)$. As can be seen from Figure 3, the HPS takes period halving exit from chaos and period doubling limit cycle route to chaos. Furthermore an in-depth observation of Figure 3(a) gives a clue of hysteresis and bistability existence by showing discontinuous bifurcation.

2.2. Multistability in HPS. Multistability in mechanical systems is important in analyzing the system performance and its nonlinear behavior. A model of a vibroimpact oscillator, known as impact pair, composed of a point mass free to move inside a periodically driven box is investigated for its multistable property [13]. The statistical mechanics of shape transitions in small elastic elliptical plates and shells driven by noise and their multistable feature is analyzed in [14]. Multistability and controllability of multistability in the presence of noise and transition to chaos in noise and quasi-periodically driven systems are of significance [15-17]. Multistable features of HPS are investigated in this section and the bifurcation diagram is obtained by plotting local maxima of the coordinate $x_{2}$ in terms of the parameter that is increased (or decreased) in tiny steps in the range of $9.8 \leq h \leq 12.2$. This strategy, known as forward and backward continuation, represents a simple way to localize the window in which the system develops multistability. The existence of multistability can be confirmed by comparing the forward (Figure 4(a) red) and backward (Figure 4(a) black) bifurcation diagrams. Figure 4(b) shows the coexisting attractors for various values of $h$ and initial conditions $[3.4,-2.1,0]$ (red plot), $[3.4,-2.1,-1.8]$ (blue plot).

\section{Time Delayed HPS (TDHPS)}

Time delayed differential equations play an important role in most of the engineering applications [18]. Time delay effects on mechanical systems have gained interest in recent years. 


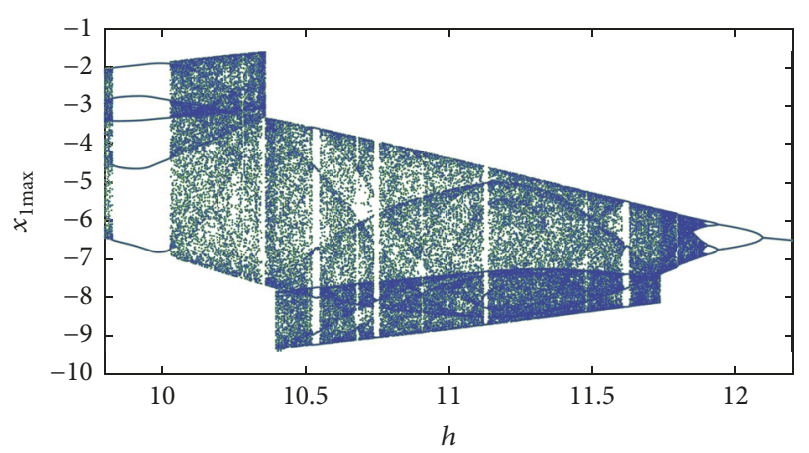

(a)

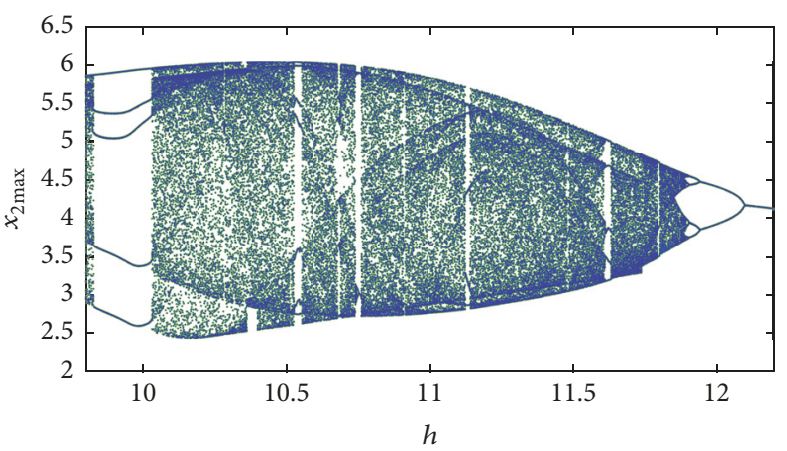

(b)

FIGURE 3: Bifurcation of the HPS (9) with the parameter $h\left((\mathrm{a}) x_{1}\right.$ and (b) $x_{2}$ ).

The increased efficiency requirements in the control inputs and controller feedback have demanded the analysis of the effect of time delays on the system. The time delays in both controllers and actuators have become a serious problem and, for instance, all digital controllers, analogue antialiasing, and reconstruction filters exhibit a certain time delay during operation, and the hydraulic actuators and human machine interaction usually show even more significant time delays [19]. Considering these facts, we are interested in analyzing the time delay effects on the HPS dynamics.

3.1. Time Delayed Nonautonomous HPS. We first introduce feedback state delay for the state variable $x_{1}$ and modify the nonautonomous HPS (8) as

$$
\begin{aligned}
\dot{x}_{1}(t)= & x_{2}(t) \\
\dot{x}_{2}(t)= & -a_{\tau} x_{2}(t)-b_{\tau} \sin x_{1}(t-\tau) \\
& +l_{\tau} \cos x_{1}(t-\tau) \sin x_{1}(t-\tau) \\
& +h_{\tau} \cos \omega_{\tau} t .
\end{aligned}
$$

and for the constant time delay $\tau=0.1$, parameter values $a_{\tau}=1.333, b_{\tau}=3.776, l_{\tau}=4.6 \times 10^{-6}, h_{\tau}=11.333$, $\omega_{\tau}=2$, and initial conditions $[-3.4,2.1,0]$, the time delayed nonautonomous HPS (TDNHPS) shows chaotic behavior as shown in Figure 5.

As discussed in the HPS, the TDNHPS also does not have a fixed point as the system is nonautonomous. To calculate the Lyapunov exponents of the TDNHPS, there are several algorithms proposed in the literature [20-22]. In this paper we adopted the technique employing the synchronization of identical systems coupled by linear negative feedback mechanism [21] for finding the exact Lyapunov exponents that are calculated as $L_{1}=0.2311, L_{2}=-1.5245$.

To analyze the impact of the time delays on the proposed TDNHPS, we derive the bifurcation of the system with the time delay $\tau$ varied between $[0.0001,1]$ and the system parameters fixed at their respective values for chaotic behavior, and the initial condition for first iteration is $[-3.4,2.1,0]$ and is changed to the end values of the state trajectory in every iteration. Figure 6 shows the bifurcation plot of TDNHPS with time delay, and as can be seen from the figure the system shows multiple chaotic regions for $0.0001 \leq \tau \leq 0.01,0.15 \leq$ $\tau \leq 0.35,0.4 \leq \tau \leq 0.72$, and $0.77 \leq \tau \leq 0.9$. The TDNHPS takes period doubling limit cycle route to chaos and inverse period doubling exit from chaos.

Figure 7 shows the bifurcation of the TDNHPS with the angular frequency of the excitation. As seen from the figure the TDNHPS shows discontinuous bifurcation with chaotic oscillations for $1.77 \leq \omega_{\tau} \leq 2.05,2.35 \leq \omega_{\tau} \leq 2.57$, and $2.6 \leq \omega_{\tau} \leq 2.7$ and takes period doubling route to chaos and period halving exit from chaos.

3.2. Time Delayed Autonomous HPS (TDAHPS). The time delayed autonomous system for the HPS (9) is derived by introducing the constant time delay in the feedback of state variable $x_{1}$ given as

$$
\begin{aligned}
\dot{x}_{1}(t)= & x_{2}(t) \\
\dot{x}_{2}(t)= & -a x_{2}(t-\tau)-b \sin x_{1}(t-\tau) \\
& +l \cos x_{1}(t-\tau) \sin x_{1}(t-\tau) \\
& +h \cos x_{3}(t) \\
\dot{x}_{3}(t)= & \omega .
\end{aligned}
$$

Like TDNHPS, the TDAHPS also shows chaotic behavior for the same conditions and parameter values, and the chaotic attractor is shown in Figure 8.

There are no defined fixed points for the system and the Lyapunov exponents of the system are calculated as $L_{1}=0.2392, L_{2}=0, L_{3}=-1.5137$. The bifurcation of the TDAHPS with time delay is given in Figure 9. Unlike TDNHPS (Figure 6), the TDAHPS shows nearly continuous bifurcation for $0.05 \leq \tau \leq 0.8$ and takes routing entry to and exit from chaos with period doubling and period halving limit cycle, respectively. Also there is significant difference between the bifurcation patterns of TDNHPS and TDAHPS confirming that the approximation of the time with a third state is not an effective method to convert nonautonomous to autonomous systems when time delay effects are considered. Similarly the effect of the parameter $\left(\omega_{\tau}\right)$ also varies between autonomous and nonautonomous HPS as can be seen from Figures 10 and 7, respectively. 


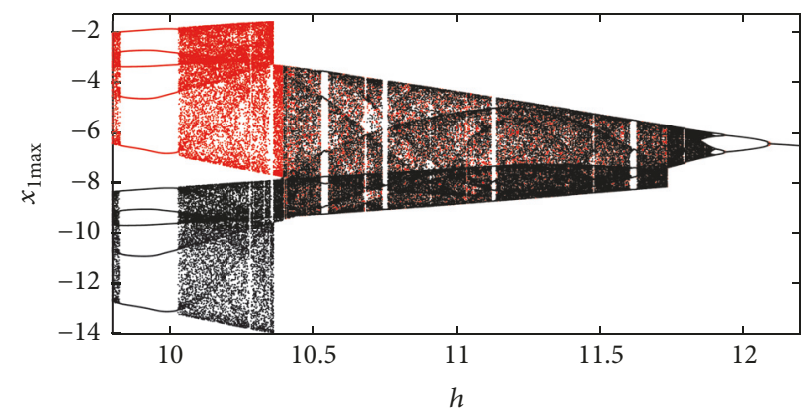

(a) Multistability in HPS with the red plots showing the forward continuation (increase parameter $h$ from 9.8 to 12.2) and black plots showing backward continuation (decrease parameter $h$ from 12.2 to 9.8 ) with reinitializing of the initial conditions

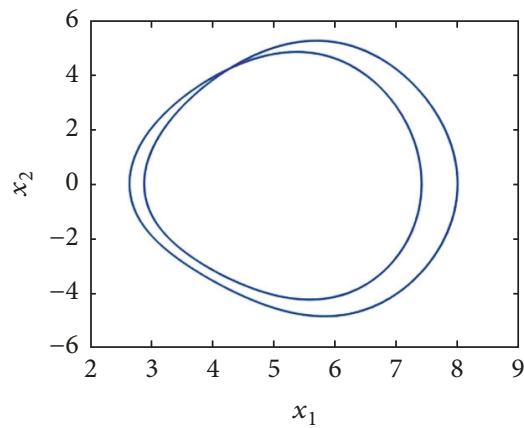

(A)

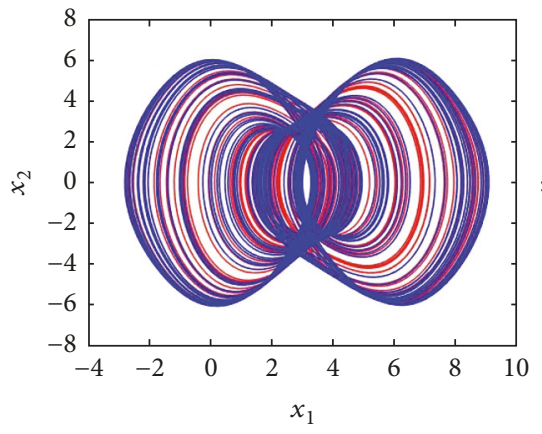

(D)

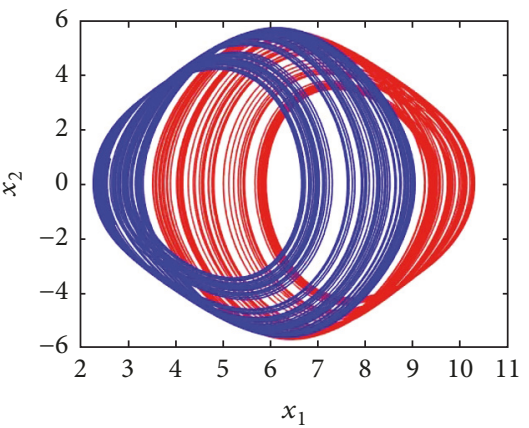

(B)

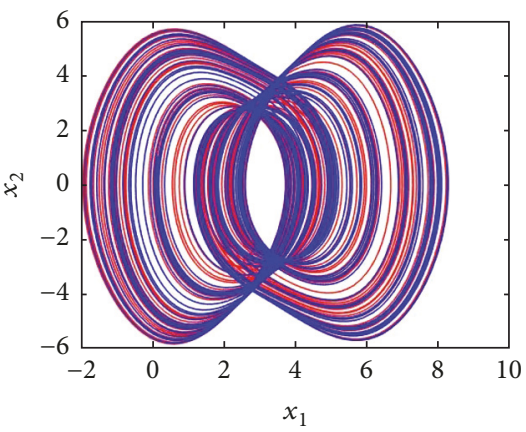

(E)

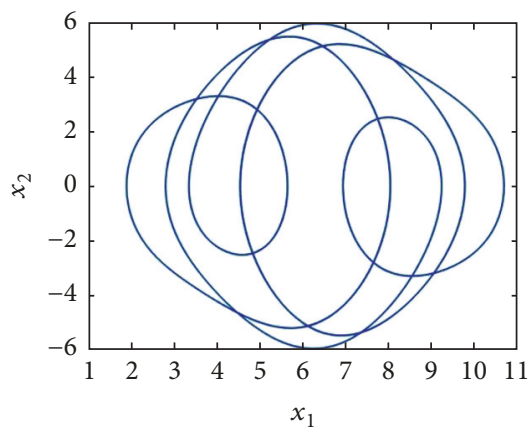

(C)

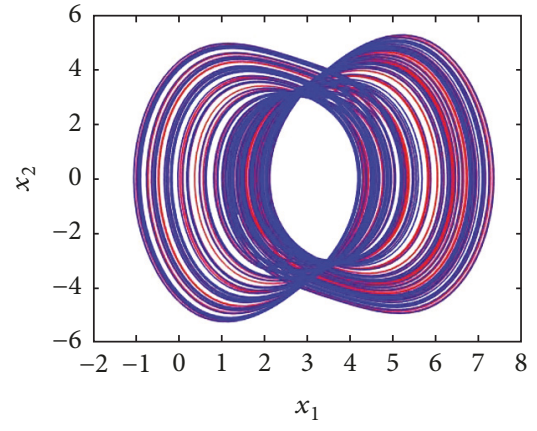

(F)

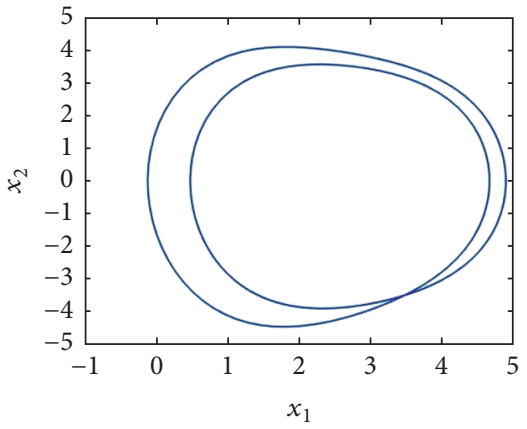

$(\mathrm{G})$

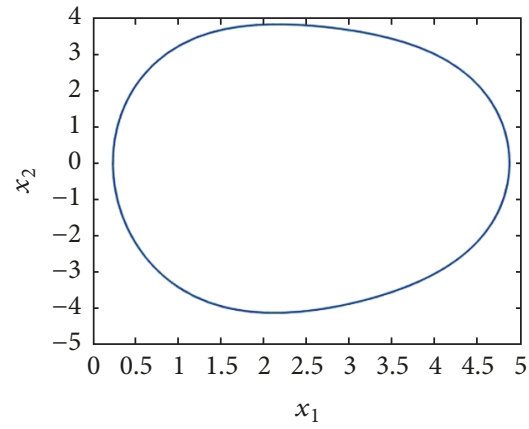

(H)

(b) Coexisting attractors for various values of $h$ and initial conditions $[3.4,-2.1,0]$ (red plot), $[3.4,-2.1,-1.8]$ (blue plot); (A) $h=9$; (B) $h=9.5$; (C) $h=10$; (D) $h=10.5$; (E) $h=11$; (F) $h=11.5$; (G) $h=12$; (H) $h=12.2$ 


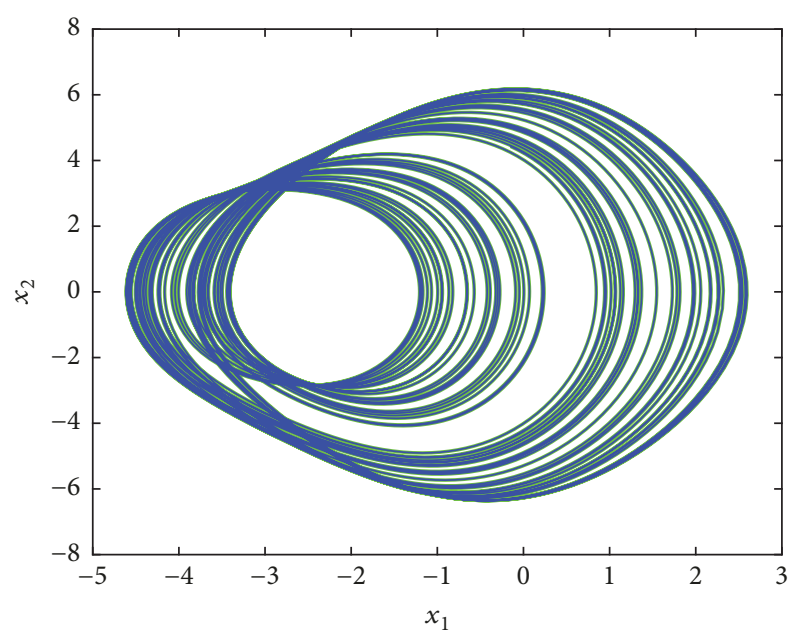

FIGURE 5: Chaotic attractor of the time delayed nonautonomous HPS.

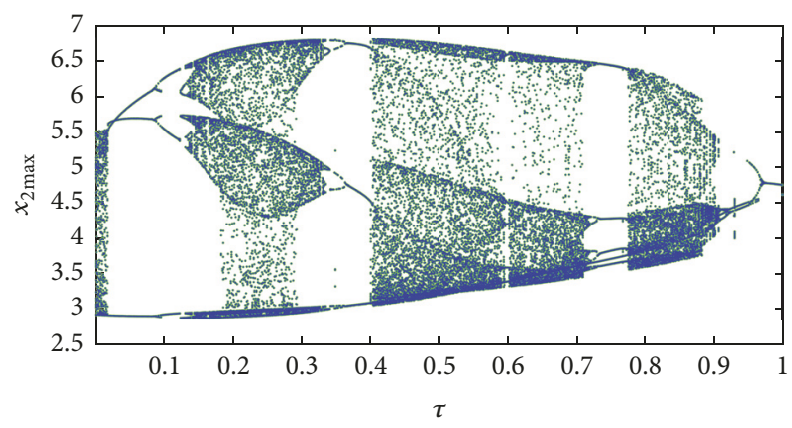

FIGURE 6: Bifurcation of the TDNHPS with time delay $\tau$.

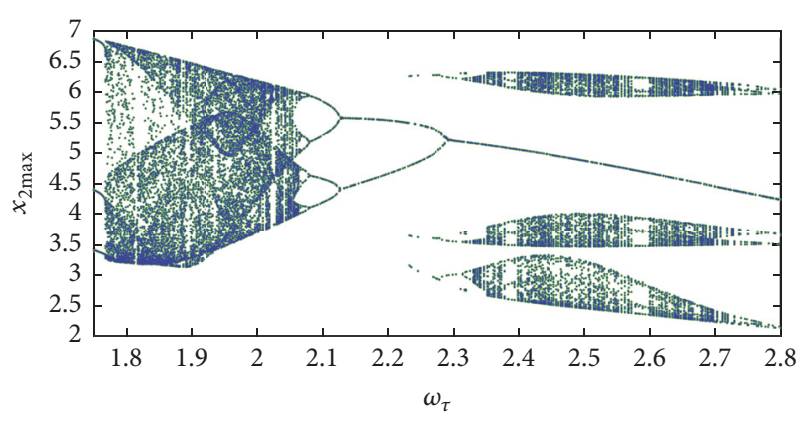

FIGURE 7: Bifurcation of the TDNHPS with parameter $\omega_{\tau}$.

3.3. Multistability in Time Delayed Autonomous HPS. To analyze the multistability existence in time delayed autonomous HPS, we derive and analyze the bifurcation of TDAHPS with parameter $h_{\tau}$. The initial condition for the first iteration is taken as $[-3.4,2.1,0]$ and is reinitialized to the last value of the state variables at the end of every iteration, plotting local maxima of the coordinate $x_{2}$ in terms of the parameter $h_{\tau}$ that is increased from 9.8 to 13 (forward continuation) and decreased from 13 to 9.8 (backward continuation) in tiny steps. The constant time delay of the TDAHPS is fixed at 0.1 . Figure 11 shows the multistability in TDAHPS, and as seen from the figure there are coexisting attractors for

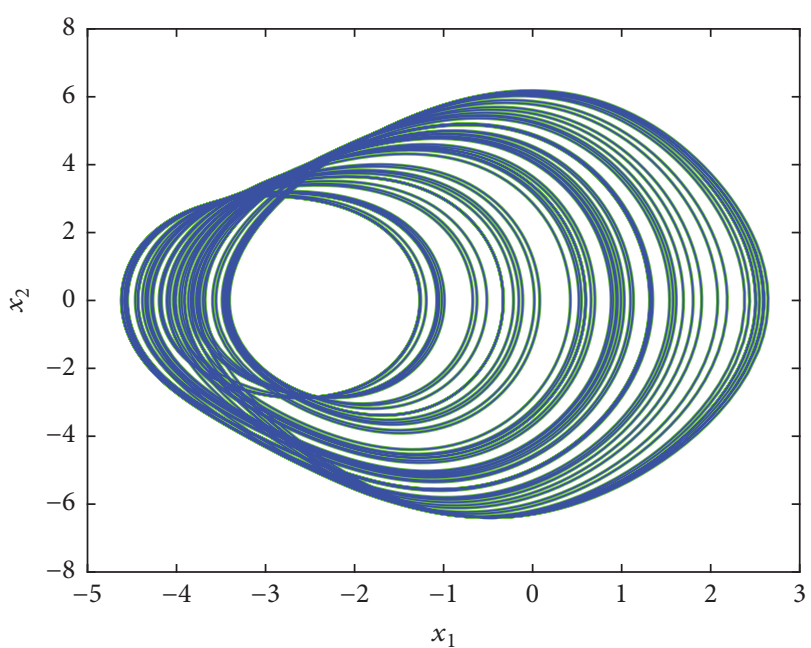

FIgURE 8: Phase portrait of the time delayed autonomous HPS.

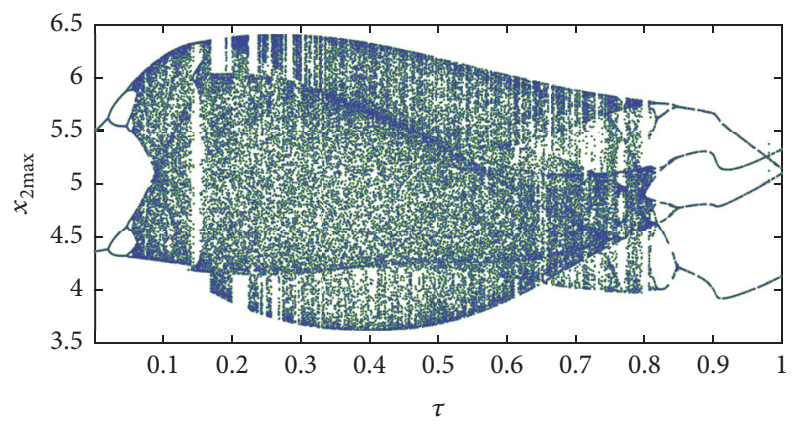

FIGURE 9: Bifurcation of the TDAHPS with time delay $\tau$.

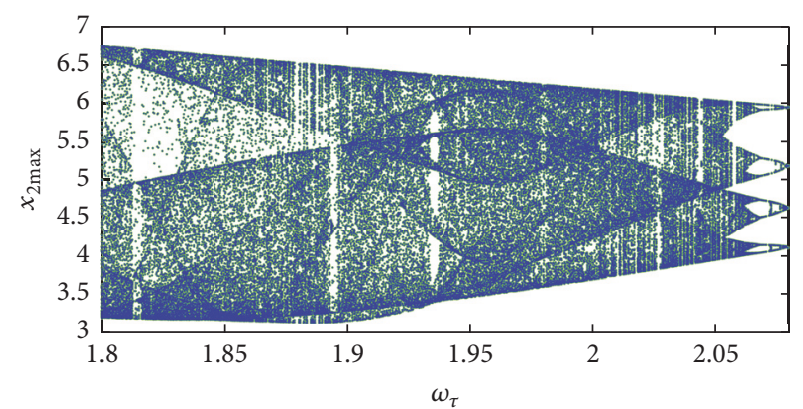

FIGURE 10: Bifurcation of the TDAHPS with parameter $\omega_{\tau}$.

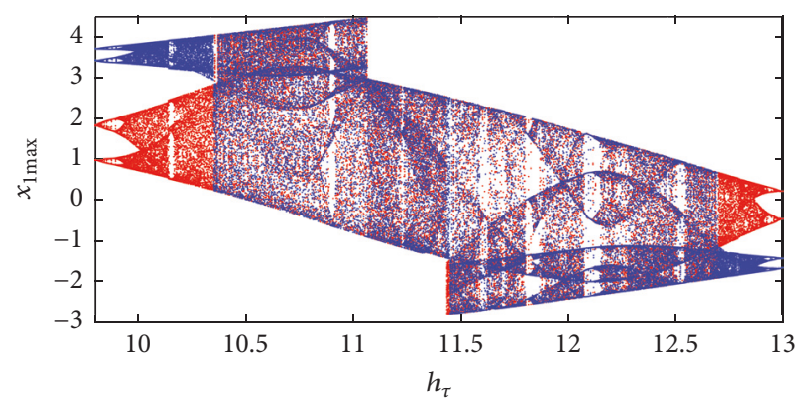

FIGURE 11: Multistability in TDAHPS with the red plots showing the forward continuation (increase in parameter $h_{\tau}$ from 9.8 to 13) and blue plots showing backward continuation (decrease in parameter $h_{\tau}$ from 13 to 9.8 ) with reinitializing of the initial conditions. 


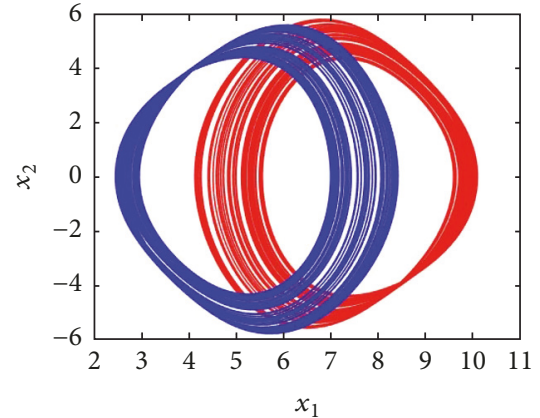

(a)

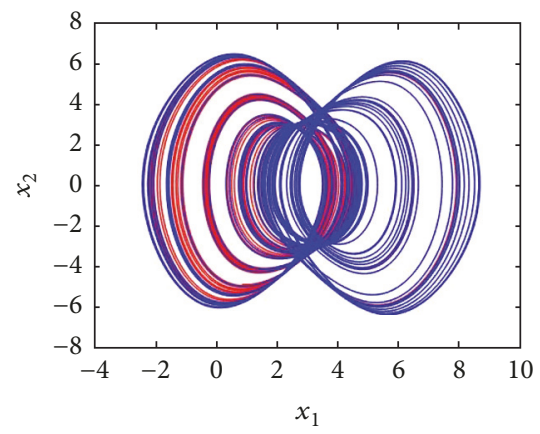

(d)

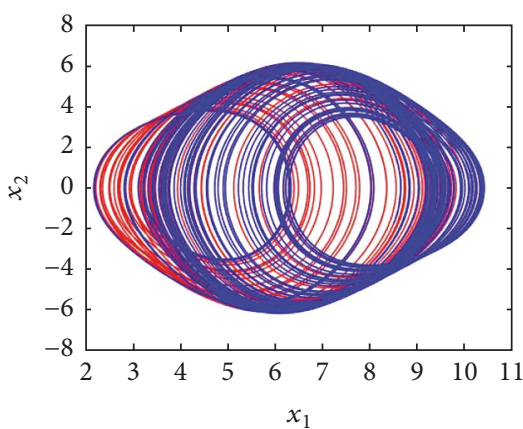

(b)

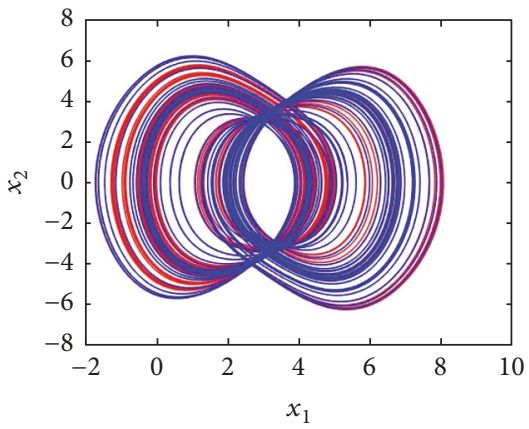

(e)

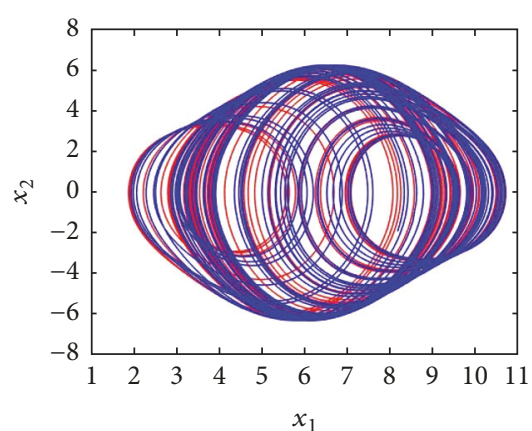

(c)

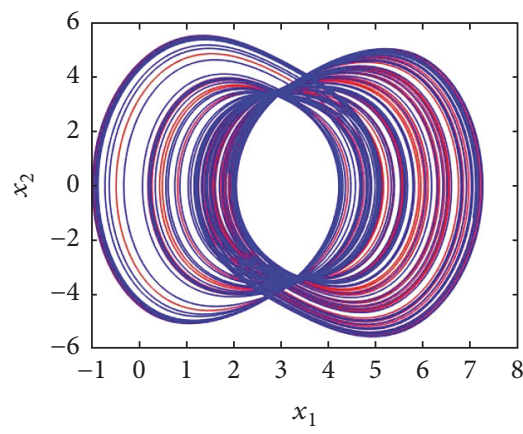

(f)

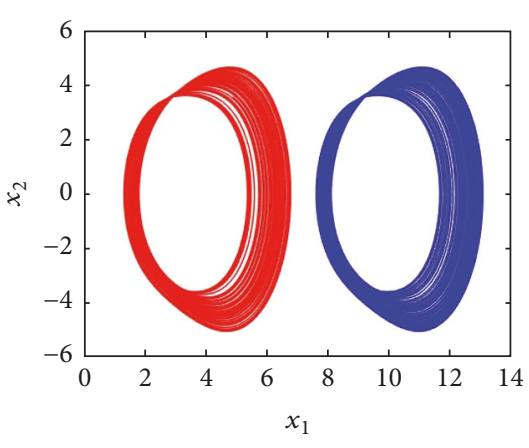

(g)

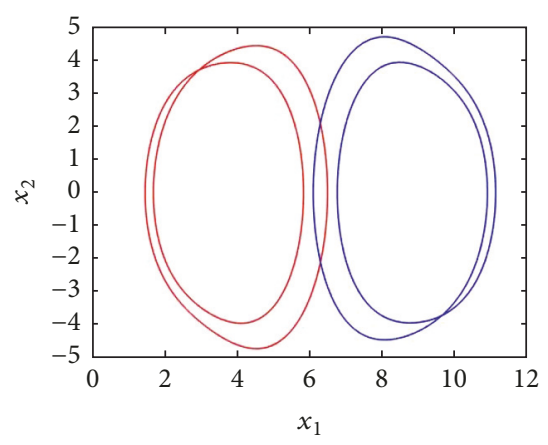

(h)

FIGURE 12: Coexisting attractors of the TDAHPS for various values of parameter $h_{\tau}$. (a) $h_{\tau}=10$; (b) $h_{\tau}=10.5$; (c) $h_{\tau}=11$; (d) $h_{\tau}=11.5$; (e) $h_{\tau}=12 ;(\mathrm{f}) h_{\tau}=12.5 ;$ (g) $h_{\tau}=12.8 ;(\mathrm{h}) h_{\tau}=13$.

$9.918 \leq h_{\tau} \leq 10.36,11.45 \leq h_{\tau} \leq 11.46$, and $12.69 \leq$ $h_{\tau} \leq 12.93$. These coexisting attractors are evidence of multistability and are seen by comparing the forward (red) and backward (blue) continuation plots. Figure 12 shows the $2 \mathrm{D}$ phase portraits of the TDAHPS.

\section{Conclusion}

Bifurcation analysis and chaotic behaviors exhibited by a horizontal platform system are studied for nonautonomous nondelayed system. The bifurcation of the HPS with parameter $h$ shows that the system shows multistability with coexisting attractors. Time delay effects on the autonomous and nonautonomous HPS are derived by introducing a constant delay with the state feedback variable $x_{1}$. Bifurcation of the autonomous and nonautonomous TDHPS with time delay shows that the system behavior differs with the introduction of the third state variable. Similarly the bifurcation of the parameters of the autonomous and nonautonomous TDHPS also shows difference. This clearly shows that a constant time delay can change the system behavior between the autonomous and nonautonomous systems. Multistability and coexisting attractors are seen in the TDHPS as well as the HPS.

\section{Conflicts of Interest}

The authors declare that there are no conflicts of interest regarding the publication of this paper.

\section{References}

[1] S. Vaidyanathan and C. Volos, Eds., Advances and applications in chaotic systems, vol. 636 of Studies in Computational Intelligence, Springer, [Cham], 2016. 
[2] K. Rajagopal, A. Karthikeyan, and P. Duraisamy, "Bifurcation Analysis and Chaos Control of a Fractional Order Portal Frame with Nonideal Loading Using Adaptive Sliding Mode Control," Shock and Vibration, vol. 2017, Article ID 2321060, 2017.

[3] P. Sekar and S. Narayanan, "Chaos in mechanical systems A review," SADHANA - Academy Proceedings in Engineering Sciences, vol. 20, no. 2-4, pp. 529-582, 1995.

[4] N.-S. Pai and H.-T. Yau, "Suppression of chaotic behavior in horizontal platform systems based on an adaptive sliding mode control scheme," Communications in Nonlinear Science and Numerical Simulation, vol. 16, no. 1, pp. 133-143, 2011.

[5] C. C. Wang, N. S. Pai, H. T. Yau et al., "Bifurcation Analysis of Horizontal Platform System," World Academy of Science, Engineering and Technology International Journal of Mathematical, Computational, Physical, Electrical and Computer Engineering, vol. 4, no. 5, 2010.

[6] M. P. Aghababa and H. P. Aghababa, "Synchronization of mechanical horizontal platform systems in finite time," Applied Mathematical Modelling, vol. 36, no. 10, pp. 4579-4591, 2012.

[7] M. P. Aghababa, "Chaotic behavior in fractional-order horizontal platform systems and its suppression using a fractional finite-time control strategy," Journal of Mechanical Science and Technology, vol. 28, no. 5, pp. 1875-1880, 2014.

[8] C. Li, Z. Gong, D. Qian, and Y. Chen, "On the bound of the Lyapunov exponents for the fractional differential systems," Chaos: An Interdisciplinary Journal of Nonlinear Science, vol. 20, no. 1, Article ID 016001CHA, 2010.

[9] A. Wolf, J. B. Swift, H. L. Swinney, and J. A. Vastano, "Determining Lyapunov exponents from a time series," Physica D: Nonlinear Phenomena, vol. 16, no. 3, pp. 285-317, 1985.

[10] S. Ellner, A. R. Gallant, D. McCaffrey, and D. Nychka, "Convergence rates and data requirements for Jacobian-based estimates of Lyapunov exponents from data," Physics Letters A, vol. 153, no. 6-7, pp. 357-363, 1991.

[11] A. Maus and J. C. Sprott, "Evaluating Lyapunov exponent spectra with neural networks," Chaos, Solitons \& Fractals, vol. 51, pp. 13-21, 2013.

[12] M. S. Tavazoei and M. Haeri, "Unreliability of frequencydomain approximation in recognising chaos in fractional-order systems," IET Signal Processing, vol. 1, no. 4, pp. 171-181, 2007.

[13] E. S. Medeiros, S. L. T. de Souza, and I. L. Caldas, "Multistability in Systems with Impacts, International conference on Chaos and Nonlinear Dynamics," in Proceedings of the Multistability in Systems with Impacts, International conference on Chaos and Nonlinear Dynamics, 2010.

[14] L. Mahadevan and L. Ee Hou Yong, "Statistical Mechanics of Multistability in Microscopic Shells".

[15] U. Feudel and C. Grebogi, "Multistability and the control of complexity," Chaos: An Interdisciplinary Journal of Nonlinear Science, vol. 7, no. 4, pp. 597-604, 1997.

[16] S. Kraut, U. Feudel, and C. Grebogi, "Preference of attractors in noisy multistable systems," Physical Review E, vol. 59, pp. 52535260, 1999.

[17] S. Kraut and U. Feudel, "Multistability, noise, and attractor hopping: The crucial role of chaotic saddles," Physical Review E: Statistical, Nonlinear, and Soft Matter Physics, vol. 66, no. 1, Article ID 015207, pp. 015207/1-015207/4, 2002.

[18] J.-P. Richard, “Time-delay systems: An overview of some recent advances and open problems," Automatica, vol. 39, no. 10, pp. 1667-1694, 2003.
[19] H. Y. Hu and Z. H. Wang, Dynamics of controlled mechanical systems with delayed feedback, Springer Berlin Heidelberg, 2002.

[20] A. Stefanski and A. Dabrowski, “Tomasz Kapitaniak, Evaluation of the largest Lyapunov exponent in dynamical systems with time delay, Chaos," Solitons Fractals, vol. 23, no. 5, pp. 1651-1659, March 2005.

[21] A. Stefanski, T. Kapitaniak, A. Dabrowski, A. Stefański, and A. Dąbrowski, "The Largest Lyapunov Exponent of Dynamical Systems with Time Delay," in Proceedings of the IUTAM Symposium on Chaotic Dynamics and Control of Systems and Processes in Mechanics, G. Rega and F. Vestroni, Eds., vol. 122, p. 122, Springer, Dordrecht, the Netherlands, 2005.

[22] A. Dabrowski, "Estimation of the largest Lyapunov exponentlike (LLEL) stability measure parameter from the perturbation vector and its derivative dot product (part 2) experiment simulation," Nonlinear Dynamics, vol. 78, no. 3, pp. 1601-1608, 2014. 


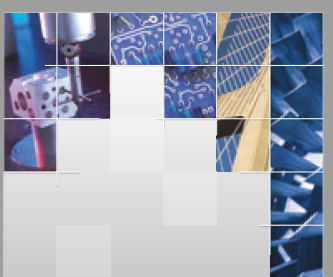

\section{Enfincering}
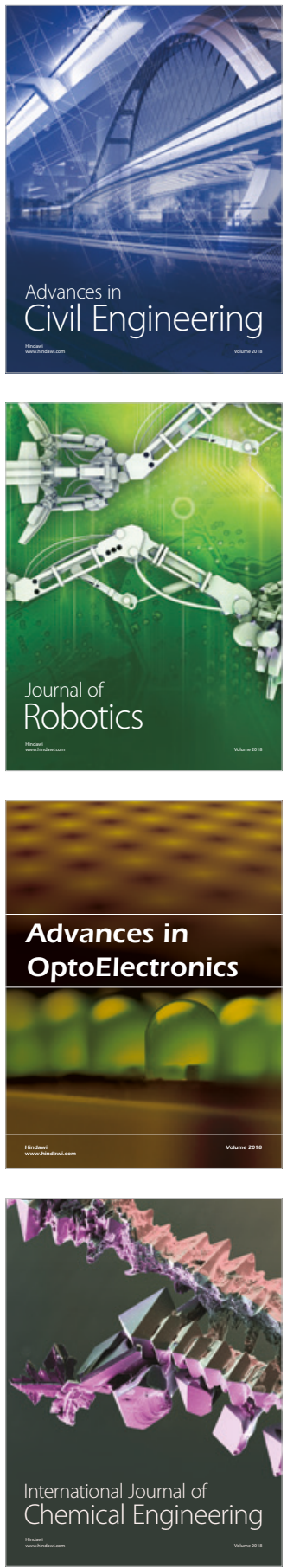

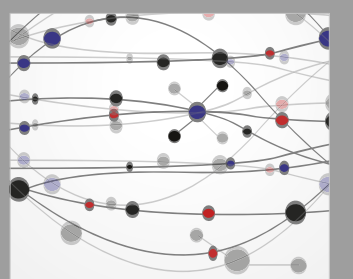

\section{Rotating \\ Machinery}

The Scientific World Journal

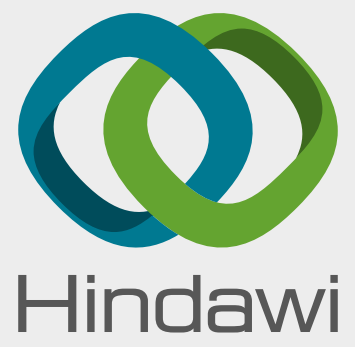

Submit your manuscripts at

www.hindawi.com
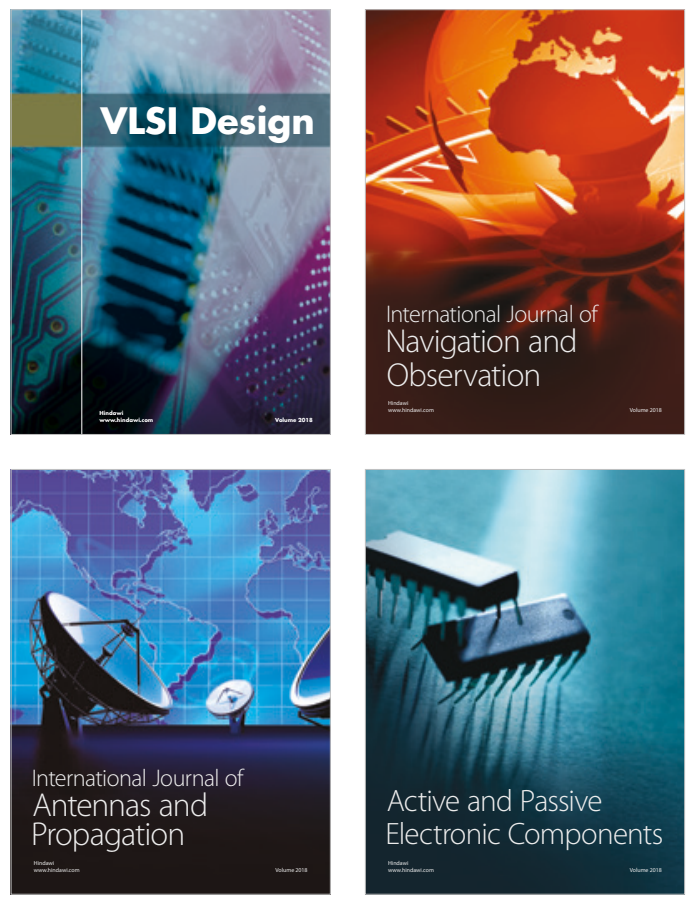
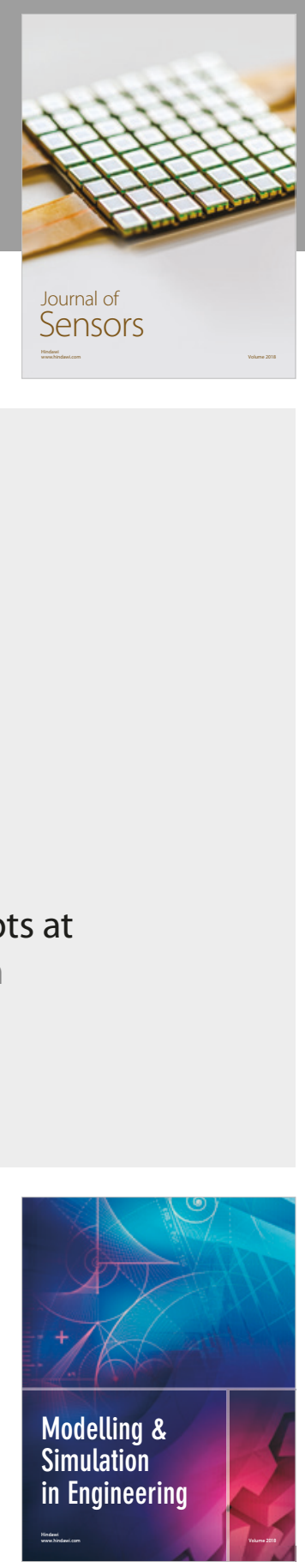

\section{Advances \\ Multimedia}
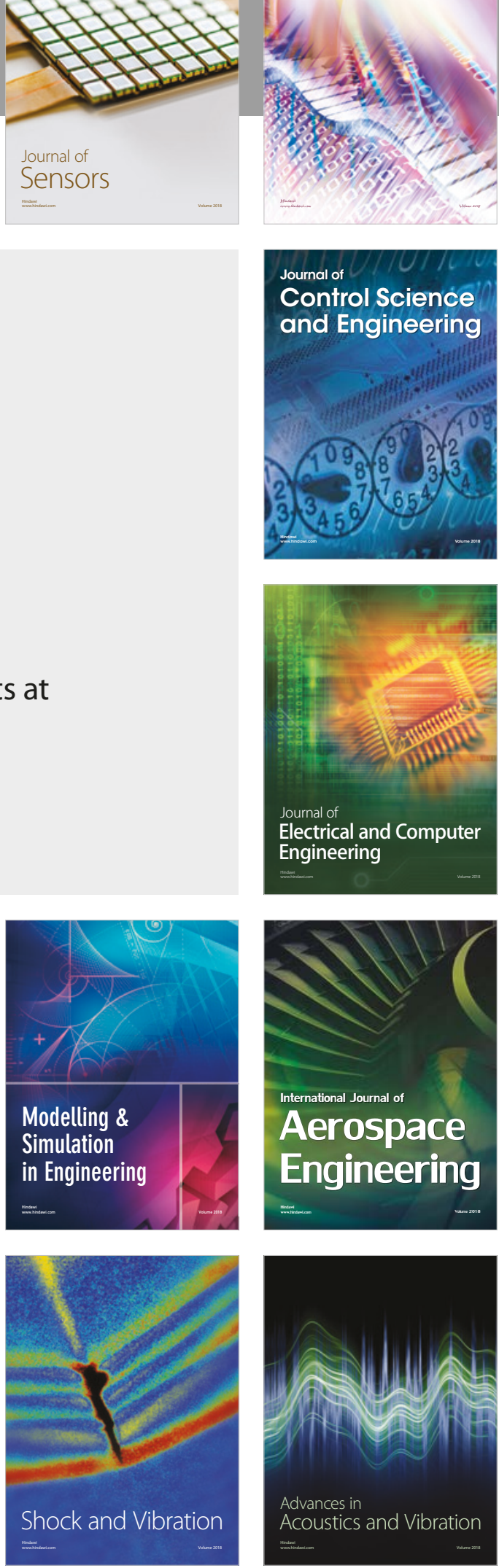